University of Nebraska - Lincoln

DigitalCommons@University of Nebraska - Lincoln

$1-1993$

\title{
Electric-field stabilization and competition of gratings in a photorefractive polymer
}

\author{
Stephen Ducharme \\ University of Nebraska-Lincoln, sducharme1@unl.edu \\ Brian Jones \\ University of Nebraska-Lincoln \\ James M. Takacs \\ University of Nebraska-Lincoln, jtakacs1@unl.edu \\ Lei Zhang \\ University of Nebraska-Lincoln
}

Follow this and additional works at: https://digitalcommons.unl.edu/physicsducharme

Part of the Physics Commons

Ducharme, Stephen; Jones, Brian; Takacs, James M.; and Zhang, Lei, "Electric-field stabilization and competition of gratings in a photorefractive polymer" (1993). Stephen Ducharme Publications. 79. https://digitalcommons.unl.edu/physicsducharme/79

This Article is brought to you for free and open access by the Research Papers in Physics and Astronomy at DigitalCommons@University of Nebraska - Lincoln. It has been accepted for inclusion in Stephen Ducharme Publications by an authorized administrator of DigitalCommons@University of Nebraska - Lincoln. 


\title{
Electric-field stabilization and competition of gratings in a photorefractive polymer
}

\author{
Stephen Ducharme and Brian Jones \\ Department of Physics and Astronomy, Center for Materials Research and Analysis, University of Nebraska, Lincoln, Nebraska 68588-0111
}

James M. Takacs and Lei Zhang

Department of Chemistry, Center for Materials Research and Analysis, University of Nebraska, Lincoln, Nebraska 68588-0304

Received September 4, 1992

\begin{abstract}
We report observations of electric-field stabilization of gratings and grating competition in a new photorefractive polymer mixture composed of $40 \mathrm{wt}$. \% diethylamino-benzaldehyde-diphenyl hydrazone dissolved in Bisphenol A $4,4^{\prime}$-nitroaminotolane. Gratings formed with a 650 -nm optical wavelength in $145-\mu \mathrm{m}$-thick films at $138-\mathrm{kV} / \mathrm{cm}$ bias field have a diffraction efficiency as high as $0.11 \%$, require an energy density of $\sim 1 \mathrm{~J} / \mathrm{cm}^{2}$ for formation, and show cancellation, revelation, and electric-field-stabilized persistence. We propose a model in which fast recording is associated with one set of photosensitive traps, whereas a slower cancellation and subsequent revelation are associated with a competing grating formed in a set of insensitive shallow traps. The model also accounts for observed fatigue of the photoconductivity.
\end{abstract}

Promising new photorefractive polymers have been reported recently. ${ }^{1-3}$ These hybrid materials combine the best available electro-optic polymers ${ }^{4}$ with charge-transport agents and (potentially) photosensitizing dyes used in electrophotography and may replace photorefractive crystals ${ }^{5}$ in future applications because of the relative low cost and flexibility of fabrication of polymers. It is necessary, however, to improve the performance of current photorefractive polymers.

We report the properties of a new photorefractive polymer that has high photorefractive sensitivity and a persistent storage mechanism stabilized by an external electric field. The new material is a mixture of the electro-optic polymer Bisphenol A 4,4'-nitroaminotolane [BisA-NAT (Ref. 4)] and 40 wt. \% diethylamino-benzaldehyde-diphenyl hydrazone [DEH (Refs. 6 and 7)], a hole transport agent incorporated to carry holes that are photogenerated from the NAT chromophore. ${ }^{1,2}$ The polymer exhibits necessary electro-optic and photoconductive response and unique features characteristic of photorefractive grating formation, readout, and erasure. The grating dynamics and corresponding fatigue of the bulk photoconduction are consistent with the presence of at least two distinct types of traps. Type A traps are highly photosensitive and are associated with the NAT chromophore. Type B traps, of unknown origin, have low photogeneration efficiencies and collect charge liberated from the type A traps. The two most striking and challenging features of grating dynamics in BisA-NAT:DEH are the electric-fieldstabilized storage and the revelation of the type $B$ gratings only during uniform illumination.

Photorefractive polymer films were made as follows: A solution of Bisphenol A diglycidyl ether ${ }^{8}$ $(1.07 \mathrm{~g}, 3.14 \mathrm{mmol})$ in propylene glycol monomethyl ether $(4 \mathrm{~mL})$ was filtered to $0.2 \mu \mathrm{m}$. The chro- mophore 4,4'-nitroaminotolane $(0.75 \mathrm{~g}, 3.15 \mathrm{mmol})$, prepared by palladium-catalyzed coupling of 4nitrophenylacetylene to 4 -iodoaniline, ${ }^{9}$ was added, and the resulting mixture was heated and stirred under a nitrogen atmosphere for $8 \mathrm{~h}$ at $100^{\circ} \mathrm{C}$. The resulting polymer had weight and number average molecular weights $M_{w}=3500$ and $M_{n}=1000$, respectively, versus a polystyrene reference in tetrehydrofuran as measured by gel-phase chromatography and a $T_{g}$ of $59^{\circ} \mathrm{C}$ measured by differential scanning calorimetry. Then a solution of $25 \mathrm{mg}$ of the polymer and $16.6 \mathrm{mg}$ of $\mathrm{DEH}$ per $1 \mathrm{~mL}$ of propylene glycol monomethyl ether was filtered again to $0.2 \mu \mathrm{m}$. One milliliter of this solution was dripped slowly onto two $25 \mathrm{~mm} \times 12 \mathrm{~mm} \times 1.1 \mathrm{~mm}$ indium tin oxide-coated glass plates, which were then set on a $95^{\circ} \mathrm{C}$ hot plate and dried for $30 \mathrm{~min}$. The plates were then pressed together and cooled, with the dried polymer and $145-\mu \mathrm{m}$-thick quartz spacers sandwiched between. The samples showed slight optical scattering, had few or no bubbles, and had absorption coefficients of $118 \pm 10 \mathrm{~cm}^{-1}$ at $650 \mathrm{~nm}$. The (reversible) dielectric strength of the samples was at least $138 \mathrm{kV} / \mathrm{cm}$.

The photoconductivity $\sigma_{\mathrm{ph}}$ was measured by illuminating the samples at normal incidence with the 650-nm, 0.5-mm-diameter (FWHM) TEM To $_{00}$ output of a $\mathrm{cw}$ dye laser while monitoring the increase in current under constant electrical bias. There were no indications of space-charge buildup or injection by the electrodes in long-term measurements, in current-voltage measurements, or in field-reversal tests. Sample heating with total power $P \leq 25 \mathrm{~mW}$ had no measurable effect on the current, as confirmed by the absence of an increase in current during long-term illumination with 514.5-nm (strongly absorbed) light.

The photosensitivity, or photoconductivity per unit intensity, $s_{\mathrm{ph}}=\sigma_{\mathrm{ph}} /$ intensity $=\Delta J / E P$ of sam- 


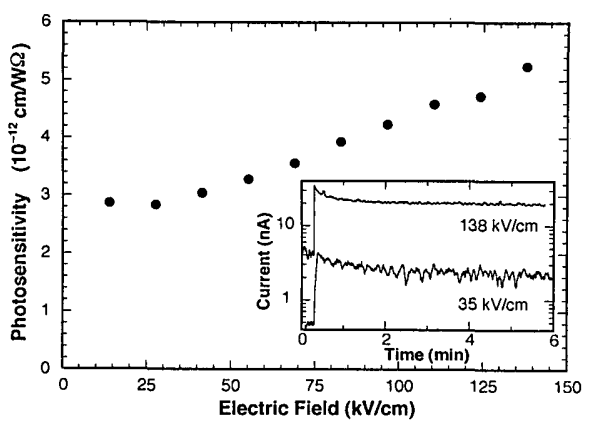

Fig. 1. Photosensitivity and photocurrent fatigue (inset) of BisA-NAT:DEH sample 220 days after fabrication.

ple 1 was $8.7 \times 10^{-11} \mathrm{~cm} / \mathrm{W} \Omega$ and of sample 2 was $1.0 \times 10^{-10} \mathrm{~cm} / \mathrm{W} \Omega$ at an electric field of $138 \mathrm{kV} / \mathrm{cm}, 650-\mathrm{nm}$ wavelength, and $100-\mathrm{mW}$ power (measured within $2 \mathrm{~h}$ after sample fabrication). Here $L$ is the sample thickness, $\Delta J$ is the photocurrent (the initial peak in the inset of Fig. 1), and $E$ is the applied electric field. These photosensitivities are approximately 100 times those reported for BisA-4-nitro-1,2-phenylenediamine (NPDA): $\mathrm{DEH}^{1}$ and 30 times larger than for polymethyl methacrylate-paranitroaniline:DEH. ${ }^{3}$ The photosensitivity increased between with field (Fig. 1), as was also observed in BisA-NPDA, ${ }^{1,2}$ likely owing to field effects on the carrier photogeneration rate and mobility. ${ }^{6,7}$

The inset of Fig. 1 shows photocurrent fatigue of approximately $30 \%$ after 30 s illumination, independent of field and intensity. This fatigue is consistent with the presence of shallow type B weakly photoionizable traps that trap approximately $30 \%$ of the charge that is photogenerated from the type A traps. The type B traps slowly trap charge until they are full, except for much lower thermal ionization and photoionization rates, which keep a small fraction of the type B traps empty. There was no observed persistence of the photocurrent, and the photosensitivity was completely restored by leaving the sample for $30 \mathrm{~min}$ or more in the dark, with no applied voltage. During this time, the shallow type B traps presumably empty into the type A traps.

Holographic measurements were made by degenerate four-wave mixing with beams originating from a $\mathrm{cw}$ dye laser operating at $650 \mathrm{~nm}$ with a single longitudinal mode and a $\mathrm{TEM}_{00}(1.0 \mathrm{~mm}$ FWHM) transverse mode. The sample was held on a stressfree mount at ambient temperature. The $s$-polarized 10 - and $9-\mathrm{mW}$ writing beams in air were incident upon the sample at $60^{\circ}$ and $40^{\circ}$ from the normal, respectively. The calculated optical fringe spacing was $2.2 \mu \mathrm{m},{ }^{10}$ and the grating wave vector was oriented at an angle of $63.6^{\circ}$ from sample normal. The $5-\mu \mathrm{W}$ $p$-polarized readout beam was present during recording and readout but had no measurable effect on the results. The Bragg nature of the diffraction was verified by rotating the sample during readout. The maximum observed diffraction efficiency was $0.11 \%$ at a bias field of $138 \mathrm{kV} / \mathrm{cm}$. The photorefractive nature of the gratings was confirmed by the unique combination of several observations ${ }^{1}$ that effectively rule out photochromic, photochemical, thermal, orientational, and other known mechanisms. The signal was observed only with the bias electric field present because the nonlinear optical chromophores were not permanently aligned, or poled, as noted above; there was no linear electro-optic response in zero field and hence no linear index grating. The holographic signal was present only when a large electric field was applied to the sample during grating formation, consistent with field-enhanced (drift) photorefractive grating formation. ${ }^{1,5}$ The gratings could be repeatedly written and completely erased (equal writing and erasure time constants) with the same optical wavelength at ambient temperature.

The main features of grating formation, cancellation, and revelation are demonstrated in Fig. 2. The type A grating was first formed by the object and reference writing beams for $2 \mathrm{~min}$, during which time a competing grating was apparently formed, followed by 2 min of waiting with only the $5-\mu \mathrm{W}$ readout beam present. The reference beam was turned back on at $5 \mathrm{~min}$ to reveal and subsequently erase the type $\mathrm{B}$ grating. The exponential rise time of the type $\mathrm{A}$ grating amplitude was $\tau=0.76 \mathrm{~s}$ at a total intensity of $I=1.5 \mathrm{~W} / \mathrm{cm}^{2}$. The measured photorefractive energy density for writing $U_{\text {meas }}=I \tau=1.1 \mathrm{~J} / \mathrm{cm}^{2}$ was significantly smaller than the expected value $U_{\text {calc }}=$ $\epsilon \epsilon_{0} / s_{\mathrm{ph}}=0.05 \mathrm{~J} / \mathrm{cm}^{2}$ (in the large grating-spacing limit ${ }^{5}$ with $\epsilon \approx 3$ ). The result that $U_{\text {calc }} \ll U_{\text {meas }}$, observed in this and other photorefractive polymers, may mean that there are parallel photoconduction channels (i.e., additional traps) that contribute to photoconduction but not to photorefractive charge storage.

After formation of the type A grating in Fig. 2, the signal began to decrease steadily with an exponential time constant of $130 \mathrm{~s}$. We attribute this decrease to cancellation by a competing type B grating. (The fluctuation of the signal during cancellation is due to fluctuation of the position of the optical interference pattern as the optical mounts shift, and hence the fast type A grating phase fluctuates relative to the phase of the slower type B grating.) Similar grating competition, screening, and fixing phenomena associated with multiple traps have all been observed in inorganic photorefractive crystals, ${ }^{11}$ and evidence for grating competition in a photorefractive polymer has recently been reported. ${ }^{3}$ The type A grating and

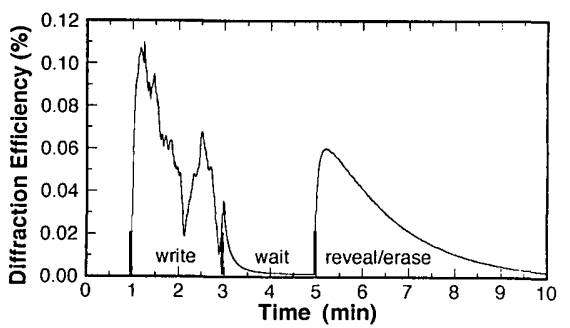

Fig. 2. Photorefractive grating diffraction efficiency with extraordinary readout polarization in sample 1 of BisA-NAT:DEH with an applied electric field of 138 $\mathrm{kV} / \mathrm{cm}$. The grating is written with a total intensity of $1.5 \mathrm{~W} / \mathrm{cm}^{2}$, ordinary polarization, and a grating spacing of $2.2 \mu \mathrm{m}$. The background was $0.0003 \%$. 


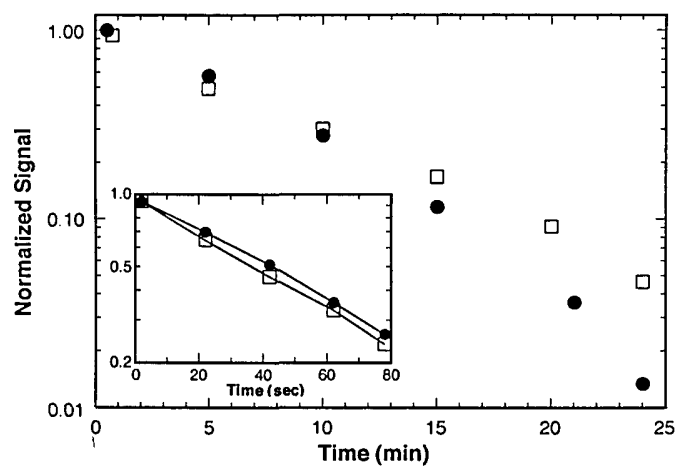

Fig. 3. Type $B$ grating erasure at $0.8-W / \mathrm{cm}^{2}$ uniform illumination and dark decay (inset) of sample 2 . The open squares are measured in zero applied field (a field was applied for $8 \mathrm{~s}$ during each measurement to align the electro-optic chromophores), and the filled circles are measured at $138 \mathrm{kV} / \mathrm{cm}$. The signal uncertainties are comparable to the size of the symbols.

the photoconductivity are associated with the NAT chromophores as was shown in BisA-NPDA:DEH., We do not know the identity of the type $B$ traps.

The type B grating signal was observed (revealed) only in the presence of sufficiently strong uniform illumination. When the writing beams were blocked, the signal dropped to $1 \%$ of its earlier strength. When only the reference beam $(10 \mathrm{~mW})$ was turned back on (see Fig. 2), the signal quickly rose to a value comparable with (and sometimes larger than) the value recorded during initial grating formation, with an exponential rise time constant of $2.6 \mathrm{~s}$ followed by a decay with a time constant of $167 \mathrm{~s}$. If the reference beam was then blocked during revealing, the signal again dropped to $1 \%$ (or less) of the initial maximum. Unblocking the reference beam again repeated the revealing process, but with a decreased peak signal.

The type B grating is stabilized by an electric field as demonstrated in Fig. 3, which shows the dark decay of the type B grating both with and without an applied field. In contrast to the decrease in decay rate of the type $B$ gratings in an electric field, the photoconductivity increases with increasing electric field (Fig. 1). Also, the light-induced erasure of the type B grating shown in the inset of Fig. 3 is the same, or perhaps slightly faster, in an electric field, suggesting that the thermal ionization path of these traps is different from the photoionization path.

We now apply the two-trap model, which is similar to the model in Ref. 12, if the shallow-trap photoionization cross section is neglected, to the present results. The highly photosensitive and thermally deep type A traps form an ordinary grating, with charge collecting in the dark parts of the interference pattern during the first few seconds of illumination. Charge collects more slowly in the thermally shallow type B traps, preferentially in the strongly illuminated regions where the photoionized charge density is highest. The type $\mathrm{B}$ traps have low photoionization sensitivity and are therefore filled with carriers that are in phase with the intensity distribution and $180^{\circ}$ out of phase with the type A charge, thus resulting in little or no net space-charge field, the cancellation observed in the 1-3 min period in Fig. 2. The type $B$ grating is revealed by uniform illumination that quickly erases the grating in the type A traps, followed by slow erasure of the type B grating. The average occupation of the type $B$ traps remains high during erasure, as in photoconduction fatigue; subsequent attempts to write gratings yield lower diffraction efficiencies owing to the depletion of the type A traps, unless the sample has been allowed to come to equilibrium for at least $30 \mathrm{~min}$ in the dark at zero voltage.

We cannot, however, explain with the two-trap model the need to illuminate the sample during readout. A more complicated model will not be meaningful with the present limited data, so we are continuing the experimental studies. Also, we have not observed photorefractive grating cancellation in BisA-NA(S)tilbene:DEH despite the close similarity between BisA-NAT:DEH and BisA-NAS:DEH in physical, chemical, optical, and photoconduction fatigue properties.

We thank C. H. Wang for helpful discussions and X. Q. Zhang for making differential scanning calorimetry and gel-phase chromatography measurements. This research was supported by the Nebraska Research Initiative through the Center for Materials Research and Analysis and by the Research Council of the University of Nebraska.

\section{References}

1. S. Ducharme, R. W. Twieg, J. C. Scott, and W. E. Moerner, Phys. Rev. Lett. 66, 1846 (1991).

2. W. E. Moerner, C. Walsh, J. C. Scott, S. Ducharme, D. M. Burland, G. C. Bjorklund, and R. J. Twieg, Proc. Soc. Photo-Opt. Instrum. Eng. 1560, 278 (1991).

3. C. A. Walsh and W. E. Moerner, J. Opt. Soc. Am. B 9, 1642 (1992).

4. D. Jungbauer, I. Teraoka, D. Y. Yoon, B. Reck, J. D. Swalen, R. Twieg, and C. G. Willson, Appl. Phys. Lett. 69, 8011 (1991).

5. P. Günter and J.-P. Huignard, Photorefractive Materials and Their Applications I: Fundamental Phenomena (Springer-Verlag, Berlin, 1988).

6. L. B. Schein, A. Rosenberg, and S. L. Rice, J. Appl. Phys. 60, 4287 (1986).

7. A. S. Diamond, Handbook of Imaging Materials (Dekker, New York, 1991).

8. Monomer-Polymer and Dajacs Laboratories, Inc., Trvose, Pa.

9. S. Takahashi, S. Kuroyama, K. Sonogashire, and N. Hagihara, Synthesis 11, 627 (1980); A. E. Steigman, E. Graham, K. J. Perry, L. R. Khundkar, L. R. Cheng, and L. T. Perry, J. Am. Chem. Soc. 113, 7658 (1991).

10. Derived by using refractive index $n=1.7$ for pure BisA-NAT from Ref. 4, although the DEH will decrease $n$. We neglect sample birefringence.

11. M. C. Bashaw, T. P. Ma, R. C. Barker, S. Mroczkowski, and R. R. Dube, Phys. Rev. B 42, 5641 (1990); D. D. Nolte, D. H. Ohlson, and A. M. Glass, Phys. Rev. Lett. 63, 891 (1989); D. Kirillov and J. Feinberg, Opt. Lett. 16, 1520 (1991)

12. P. Tayebati and D. Mahgerefteh, J. Opt. Soc. Am. B 8, 1053 (1991). 